'Departamento de Especialidades Médicas, Facultad de Medicina, Universidad de La Frontera. Temuco, Chile. ${ }^{2}$ Unidad de Neurología. Hospital Dr. Hernán Henríquez Aravena. Temuco, Chile. ${ }^{3}$ Centro de Excelencia en Capacitación, Investigación y Gestión para la Salud Basada en Evidencia (CIGES), Facultad de Medicina, Universidad de La Frontera. Temuco, Chile. ${ }^{4}$ Centro de Investigación en Epidemiología Cardiovascular y Nutricional (EPICYN), Facultad de Medicina, Universidad de La Frontera. Temuco, Chile. ${ }^{5}$ Facultad de Ciencias Médicas, Universidad de Santiago. Santiago, Chile.

Trabajo no recibió financiamiento. Los autores declaran no tener conflictos de interés.

Recibido el 27 de septiembre de 2020, aceptado el 10 de mayo de 2021.

Correspondencia a: Dr. Álvaro Soto Venegas Departamento de Especialidades Médicas, Facultad de Medicina, Universidad de La Frontera. Francisco Salazar \# 01145. Temuco, Chile. alvaro.soto@ufrontera.cl

\section{Tendencia de la mortalidad por enfermedades cerebrovasculares en Chile entre 1980 y 2015}

\author{
ÁLVARO SOTO V. ${ }^{1,2,3,4}$, JAIRO VANEGAS L. ${ }^{5}$
}

\section{Trend in stroke mortality in Chile from 1980 to 2015}

Background: Stroke is the third largest single cause of death in Chile, responsible for $7.3 \%$ of all deaths in 2019. Large declines in stroke mortality rates in most Latin American countries in recent decades have been reported. Aim: To analyze the trend in stroke mortality in Chile between 1980 and 2015. Material and Methods: We extracted data for age-standardized death rate (ASDR) stroke mortality per 100,000 inhabitants in Chile for the period 1980-2015 from the WHO Mortality Database. Joinpoint regression analysis was used to analyze the trend and compute the average annual percent change (AAPC) by gender in Chile. Results: The ASDR from stroke decreased from 92.8 per 100,000 in 1980 to 34.4 per 100,000 in 2015. The AAPC was $-2.8 \%(-3.5,-2.1)$, with two jointpoints, 2008 and 2012. By gender, the AAPC was $-2.4 \%$ and $-2.9 \%$ in men and women, respectively. Conclusions: Stroke mortality rate decreased significantly between 1980 and 2015 in Chile, mainly in women.

(Rev Med Chile 2021; 149: 554-558)

Key words: Chile; Mortality; Regression Analysis; Stroke.
L as enfermedades cerebrovasculares (ECV) constituyen la tercera causa específica de muerte en Chile, dando cuenta de 7.991 (7,3\%) fallecimientos el año $2019^{1}$.

Comparada con otros países, la tasa de mortalidad estandarizada por edad (TMEE) de ECV para el año 2015, en Chile se ubica en un nivel intermedio, similar a Colombia (42,1 por 100.000 habitantes) y Argentina (36,8 por 100.000 habitantes). Sin embargo, Chile tiene una tasa de mortalidad que duplica la de países desarrollados como Estados Unidos (21,9 por 100.000 habitantes) y Canadá (16,4 por 100.000 habitantes) ${ }^{2}$.

En las últimas décadas, se han comunicado reducciones significativas de las tasas de mortalidad por ECV, principalmente en países desarrollados $^{3,4}$. Esto ha sido atribuido a la disminución de la incidencia de ECV debido a una mejor prevención primaria y control de los factores de riesgo, así como a progresos en el manejo agudo de las ECV lo que ha conducido a una reducción de la letalidad ${ }^{3}$.

El objetivo de este estudio es analizar la tendencia de la mortalidad por ECV en Chile en el período 1980-2015.

\section{Material y Método}

Se obtuvieron las TMEE por 100.000 habitantes en Chile entre los años 1980 y 2015, desde la Base de Datos de Mortalidad de la Organización Mundial de la Salud ${ }^{5}$. Las muertes por ECV fueron registradas mediante los códigos CIE-10 I60-I69 de la Clasificación Internacional de Enfermedades versión 10. Debido a que se 
trabaja con datos públicos anonimizados, no fue necesaria la aprobación del estudio por un comité de ética.

Se utilizó el programa de regresión Joinpoint (versión 4.8.0.1; Surveillance Research Program, USA National Cancer Institute, Bethesda, MD, USA) para analizar los cambios significativos en la tendencia de la mortalidad. Este análisis permite identificar puntos de inflexión denominados "puntos de unión" (joinpoints) en los cuales se registró un cambio significativo en la pendiente lineal de la tendencia ${ }^{6}$. El número y localización de los puntos de unión significativos fue calculado usando un modelo log-lineal. Calculamos el Cambio Porcentual Anual (CPA), con su intervalo de confianza de $95 \%$, para describir la magnitud del cambio para cada tendencia identificada. En este modelo, la TMEE fue usada como la variable dependiente y el año de fallecimiento como la variable independiente. Se consideró un valor $\mathrm{p}<0,05$ para determinar la significancia estadística. También, calculamos el Cambio Porcentual Anual Promedio (CPAP) en total y por sexo para el período 1980-2015. El Análisis de Regresión Jointpoint ha sido ampliamente utilizado en estudios de tendencias en ECV y Enfermedad de Alzheimer ${ }^{3,4,7}$.

\section{Resultados}

Entre 1980 y 2015, el número de fallecimientos por ECV en Chile aumentó de 6.743 (3.165 hombres y 3.578 mujeres) a 8.480 (4.077 hombres y 4.403 mujeres). La TMEE por ECV descendió de 92,8 por 100.000 en 1980 a 34,4 por 100.000 en 2015 (Figura 1). Durante el período 1980-2015, el país mostró una disminución estadísticamente significativa en la tasa de mortalidad por ECV con un CPAP de -2,8\%, con dos puntos de unión: 2008 y 2012 (Tabla 1).

\section{Tendencia de mortalidad por sexo}

La TMEE por ECV en hombres descendió de 103,4 por 100.000 en 1980 a 42 por 100.000 en 2015 (Figura 2). Durante el período estudiado la tasa de mortalidad por ECV en hombres mostró una disminución estadísticamente significativa con un CPAP de $-2,4 \%$ sin puntos de unión (Tabla 1).

A su vez, la TMEE por ECV en mujeres descendió desde 84,9 por 100.000 en 1980 , a 28,9 por 100.000 en 2015 (Figura 2). La tasa de mortalidad por ECV en mujeres mostró una disminución estadísticamente significativa con un CPAP de $-2,9 \%$ mostrando dos puntos de unión: 1992 y 1995 (Tabla 1).

\section{Discusión}

En nuestro estudio encontramos una disminución estadísticamente significativa de la tasa de mortalidad por ECV en Chile, entre 1980 y 2015, en ambos sexos, más marcada en mujeres.

La mortalidad por ECV depende principalmente de la incidencia y la letalidad. En un estudio realizado en la ciudad de Iquique (2005), la incidencia de ECV ajustada por edad fue de 140,1 por 100.000 habitantes. La letalidad a 30 días fue de $23,3 \%$ y de $33 \%$ a los 6 meses $^{8}$. Por otra parte, en un estudio ejecutado en la Región de Nuble (2021) la incidencia de ECV ajustada por edad fue 163,4 por 100.000 habitantes. La letalidad a 30 días fue de $24,6 \%$ y de $30 \%$ a los 6 meses 9 .

Una de las principales estrategias implementa-

Tabla 1. Análisis de regresión Joinpoint de la tendencia de mortalidad por Enfermedades Cerebrovasculares en Chile, 1980-2015

\begin{tabular}{|lccccccc|}
\hline & Período total & \multicolumn{2}{c}{ Período 1} & \multicolumn{2}{c}{ Período 2 } & \multicolumn{2}{c|}{ Período 3} \\
& CPAP (IC 95\%) & Años & CPA (IC 95\%) & Años & CPA (IC 95\%) & Años & CPA (IC 95\%) \\
\hline Total & $-2,8^{*}(-3,5 ;-2,1)$ & $1980-2008$ & $-2,9^{*}(-3,1 ;-2,7)$ & $2008-2012$ & $-0,1(-5,1 ; 5,2)$ & $2012-2015$ & $-6,0^{*}(-10,7 ;-1,0)$ \\
\hline Hombres & $-2,4^{*}(-2,5 ;-2,3)$ & & & & & & \\
\hline Mujeres & $-2,9^{*}(-3,8 ;-2,0)$ & $1980-1992$ & $-2,7^{*}(-3,3 ;-2,1)$ & $1992-1995$ & $-6,5(-16,0 ; 4,0)$ & $1995-2015$ & $-2,5^{*}(-2,8 ;-2,2)$ \\
\hline
\end{tabular}

CPAP: cambio porcentual anual promedio; CPA: cambio porcentual anual; IC: intervalo de confianza. ${ }^{*} \mathrm{P}<0,05$ para cambio en la tendencia. 

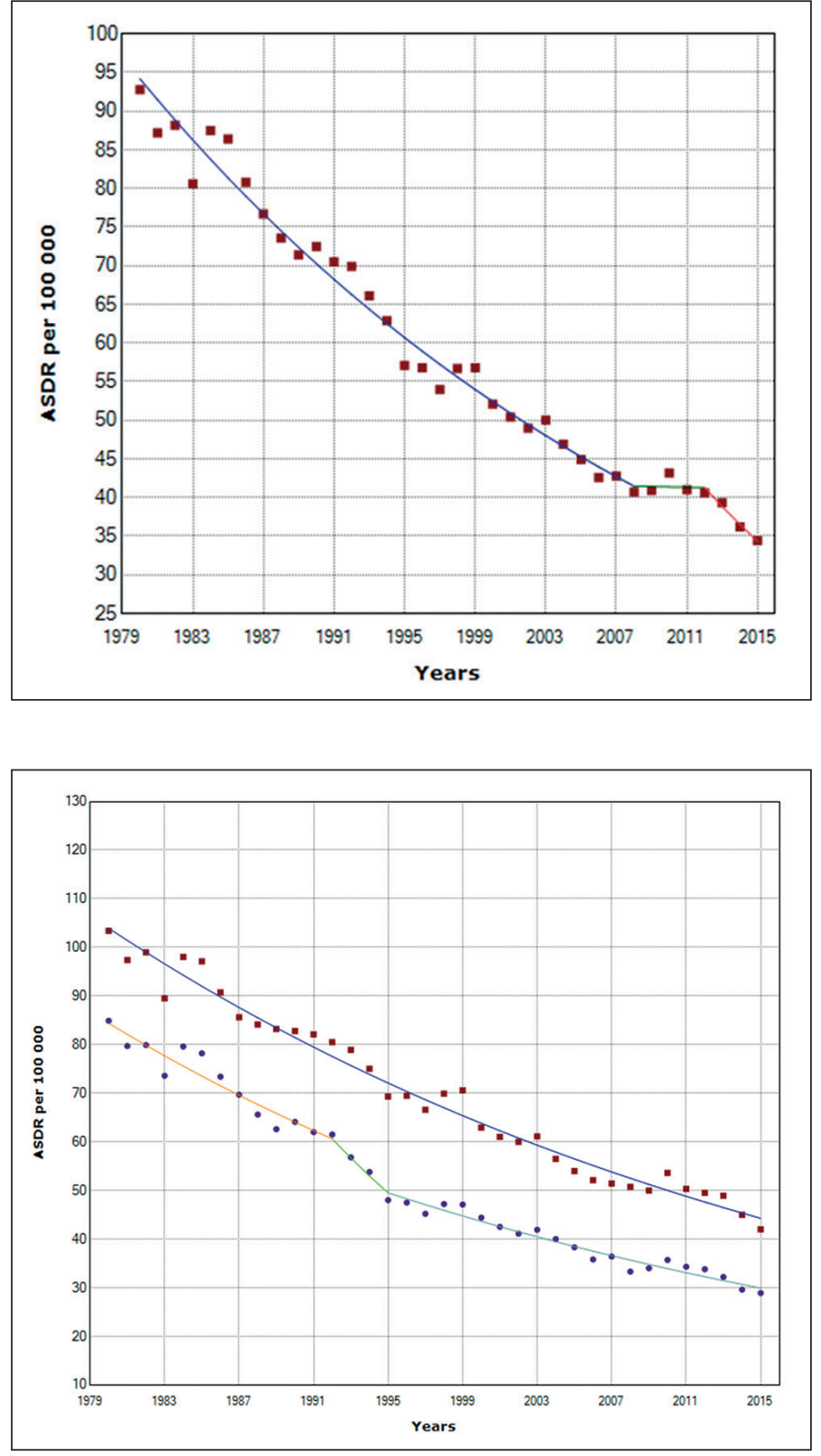

Figura 1. Tendencia de la mortalidad por Enfermedades Cerebrovasculares en Chile (total), 1980-2015.
Figura 2. Tendencia de la mortalidad por Enfermedades Cerebrovasculares en Chile, 1980-2015. Hombres $\square$, mujeres das en Chile para disminuir la morbi-mortalidad de las ECV ha sido la incorporación del ataque cerebrovascular (ACV) isquémico y la hemorragia subaranoidea aneurismática al Régimen de Garantías Explícitas en Salud (GES) en $2005^{10}$. Esto ha permitido un mayor acceso al diagnóstico con imágenes, tratamiento de las hemorragias aneurismáticas, hospitalización y prevención secundaria, además de generar Guías de Práctica Clínica basadas en la mejor evidencia disponi$b^{1}{ }^{10}$. Asimismo, desde el año 2005, otros factores de riesgo cerebrovascular como la hipertensión arterial y la diabetes, están incluidos en el GES, garantizando el acceso, oportunidad y protección financiera a los beneficiarios ${ }^{11}$. Por otra parte, la Estrategia Nacional de Salud para la década 
2011-2020 tiene como una de metas aumentar en $10 \%$ la sobrevida al primer año de los pacientes egresados con el diagnóstico de ACV. La meta es aumentar la sobrevida de 81,5 a $89,7 \%$ para el año $2020^{12}$. Estas políticas públicas se han enfocado en el control de los factores de riesgo cerebrovascular para disminuir la incidencia de ECV y en mejorar el tratamiento agudo de los pacientes con ECV para disminuir la letalidad.

La principal intervención para reducir la mortalidad y discapacidad de los pacientes con ECV es el ingreso de los pacientes a Unidades de Tratamiento de $\mathrm{ACV}^{13}$. En Chile, existen diferencias significativas en el acceso de los pacientes con ACV a estas unidades entre usuarios de los sistemas público y privado de salud ${ }^{14}$. También debemos reconocer el rol de las terapias de reperfusión, trombólisis endovenosa y trombectomía mecánica en la reducción de la mortalidad por ECV. La trombólisis se inició en Chile en clínicas privadas en 1996, y en hospitales públicos en $2009^{15}$. El efecto de la trombectomía mecánica sobre la mortalidad por ECV en el período estudiado es marginal ya que el uso de este tratamiento se ha extendido a partir de 2015.

La disminución de la mortalidad por ECV ha sido comunicada en países desarrollados. En Estados Unidos, para el período 2005-2015 la TMEE disminuyó en 21,7\% (de 48 a 37,6 por 100.000 habitantes $)^{16}$. En un estudio reciente, se comunicó una disminución de la mortalidad por ECV en la Unión Europea entre 1996 y 2015 con un CPAP de $-4,2 \%{ }^{4}$. Nuestros resultados coinciden con un estudio realizado en Brasil que encontró una disminución de 14,99\% en la mortalidad por ECV estandarizada por edad entre los años 2000 y $2009^{17}$.

Este trabajo tiene varias fortalezas. En primer lugar, según nuestro conocimiento, es el estudio más reciente que ha analizado la tendencia de la mortalidad por ECV en Chile. Por otra parte, las tasas de mortalidad fueron extraídas directamente desde una base de datos oficial. Finalmente, el análisis de regresión Jointpoint ha sido utilizado ampliamente en el estudio de tendencias de mortalidad por $\mathrm{ECV}^{3,4}$. Además, este método de análisis consigue un mejor ajuste comparado con modelos lineales, que reducen la tendencia a una sola regresión ${ }^{18}$.

Nuestro estudio también tiene algunas limitaciones. En primer lugar, la base de datos utilizada no incluía las tasas de mortalidad por tipo de ECV. En segundo lugar, no contamos con datos a nivel regional. Lo que es significativo, ya que se han comunicado diferencias significativas en la mortalidad por ECV entre regiones de Chile. En base a datos del año 2003 se reportaron tasas de mortalidad ajustada dos veces mayores para las regiones de Valparaíso, Maule, Bío-Bío y la Araucanía. El 62\% de la variabilidad de las tasas regionales ha sido atribuido a la prevalencia combinada de factores como pobreza (34\%), diabetes $(17 \%)$, sedentarismo $(8 \%)$ y sobrepeso $(3 \%)^{19}$.

Finalmente, reconocemos que los estudios de tendencias de mortalidad solo son capaces de describir tendencias y no pueden explicarlas ${ }^{3}$. Este análisis requiere ser complementado con estudios que permitan asociar la mortalidad por ECV con variables socioeconómicas como ingresos y tipo de seguro de salud entre otros determinantes sociales de la salud relevantes ${ }^{3}$.

Según el Censo 2017, la proporción de personas de 65 o más años, que es el grupo etario con mayor riesgo de ECV, alcanza $11,4 \%$, lo que es significativamente mayor al 6,6\% registrado en el Censo $1992^{20}$. Por lo tanto, a pesar de la declinación de la tasa de mortalidad por ECV, es esperable que el número absoluto de fallecimientos por ECV aumente en las próximas décadas en Chile debido al envejecimiento poblacional.

En conclusión, la tasa de mortalidad por ECV en Chile ha disminuido significativamente entre 1980 y 2015, en ambos sexos, principalmente en mujeres, lo que podría ser explicado por la reducción de la letalidad de las ECV en Chile en las últimas dos décadas.

\section{Referencias}

1. Ministerio de Salud. Estadísticas de defunciones por causa básica de muerte. Departamento de Estadísticas e Información en Salud, DEIS. Chile (2021). Disponible en: https://public.tableau.com/profile/deis4231\#!/ vizhome/DefuncionesSemanales1_0/DEF?publish=yes [Consultado el 23 de enero de 2021].

2. Organización Panamericana de la Salud. Plataforma de Información en Salud para las Américas (PLISA). Disponible en: https://www.paho.org/data/index.php/ en/indicators/visualization.html [Consultado el 23 de enero de 2021].

3. Shah R, Wilkins E, Nichols M, Kelly P, El-Sadi F, Wright FL, et al. Epidemiology report: trends in sex-specific 
cerebrovascular disease mortality in Europe based on WHO mortality data. Eur Heart J. 2019; 40 (9): 755-64.

4. Soto Á, Guillén-Grima F, Morales G, Muñoz NS, Aguinaga-Ontoso I. Trends in mortality from stroke in the European Union, 1996-2015. Eur J Neurol. 2021; 28 (1): 182-91.

5. World Health Organization Mortality Database. Disponible en: https://apps.who.int/healthinfo/statistics/ mortality/whodpms/ [Consultado el 30 de agosto de 2020].

6. Kim HJ, Fay MP, Feuer EJ, Midthune DN. Permutation tests for Joinpoint regression with application to cancer rates. Stat Med. 2000; 19: 335-51.

7. Niu H, Álvarez-Álvarez I, Guillen-Grima F, Al-Rahamneh MJ, Aguinaga-Ontoso I. Trends of mortality from Alzheimer's disease in the European Union, 1994-2013. Eur J Neurol. 2017; 24 (6): 858-66.

8. Lavados PM, Sacks C, Prina L, Escobar A, Tossi C, Araya F, et al. Incidence, 30-day case-fatality rate, and prognosis of stroke in Iquique, Chile: A 2-year community-based prospective study (PISCIS project). Lancet 2005; 365 (9478): 2206-15.

9. Lavados PM, Hoffmeister L, Moraga AM, Vejar A, Vidal C, Gajardo C, et al. Incidence, risk factors, prognosis, and health-related quality of life after stroke in a low-resource community in Chile (ÑNDU): a prospective population-based study. Lancet Glob Health 2021. https://doi.org/10.1016/S2214-109X(20)30470-8.

10. Ministerio de Salud. Plan de Acción Ataque Cerebrovascular, 2a Edición. Chile (2014). Disponible en: https:// redcronicas.minsal.cl/wp-content/uploads/2019/04/ Plan-de-accion-acv.pdf [Consultado el 27 de julio de 2020].

11. Escobar MC. Prevención del riesgo cardiovascular: políticas chilenas. Rev Med Clin Condes 2012; 23 (6): 651-5.

12. Ministerio de Salud. Estrategia Nacional de Salud: Para el cumplimiento de los Objetivos Sanitarios de la Década 2011-2020. Chile (2011). Disponible en: https://www.minsal.cl/portal/url/item/c4034eddbc96ca- 6de0400101640159b8.pdf [Consultado el 26 de julio de 2020].

13. Langhorne $\mathrm{P}$, Ramachandra S. Organised inpatient (stroke unit) care for stroke: network meta-analysis. Cochrane Database of Systematic Reviews 2020, Issue 4. Art. No.: CD000197. DOI: 10.1002/14651858. CD000197.pub4.

14. Ouriques Martins SC, Sacks C, Hacke W, Brainin M, Figueiredo F, Pontes-Neto OM, et al. Priorities to reduce the burden of stroke in Latin American countries. Lancet Neurol. 2019; 18: 674-83.

15. Figueroa-Reyes T, Sáez D, Mansilla E, Sánchez R, Nogales-Gaete J, Delgado Y. Experiencia de trombolisis sistematizada en infarto cerebral agudo en un hospital público de Chile. Rev Med Chile 2011; 139: 1118-27.

16. Benjamin EJ, Virani SS, Callaway CW, Chamberlain AM, Chang AR, Cheng S, et al. On behalf of the American Heart Association Council on Epidemiology and Prevention Statistics Committee and Stroke Statistics Subcommittee. Heart Disease and Stroke Statistics-2018 Update: A Report from the American Heart Association. Circulation 2018; 137: e67-e492. https://doi. org/10.1161/CIR.0000000000000558.

17. Garritano CR, Mendes Luz P, Lucia M, Pires E, Serrano Barbosa MT, Moreira Batista K. Analysis of the Mortality Trend due to Cerebrovascular Accident in Brazil in the XXI Century. Arq Bras Cardiol. 2012; 98 (6): 519-27.

18. Puig X, Ginebra J, Gispert R. Analysis of mortality time trend using generalized linear models. Gac Sanit. 2005; 19 (6): 481-5.

19. Lavados PM, Díaz V, Jadue L, Olavarría VV, Cárcamo DA, Delgado I. Socioeconomic and cardiovascular variables explaining regional variations in stroke mortality in Chile: An ecological study. Neuroepidemiology 2011; 37 (1): 45-51.

20. Instituto Nacional de Estadísticas. Resultados definitivos Censo 2017. Chile (2017). Disponible en: https://www. censo2017.cl/wp-content/uploads/2017/12/Presentacion_Resultados_Definitivos_Censo2017.pdf [Consultado el 21 de septiembre de 2020). 